\title{
Doença celíaca, hábitos e práticas alimentares e qualidade de vida
}

\author{
Celiac disease, eating habits and \\ practices and life quality of life
}

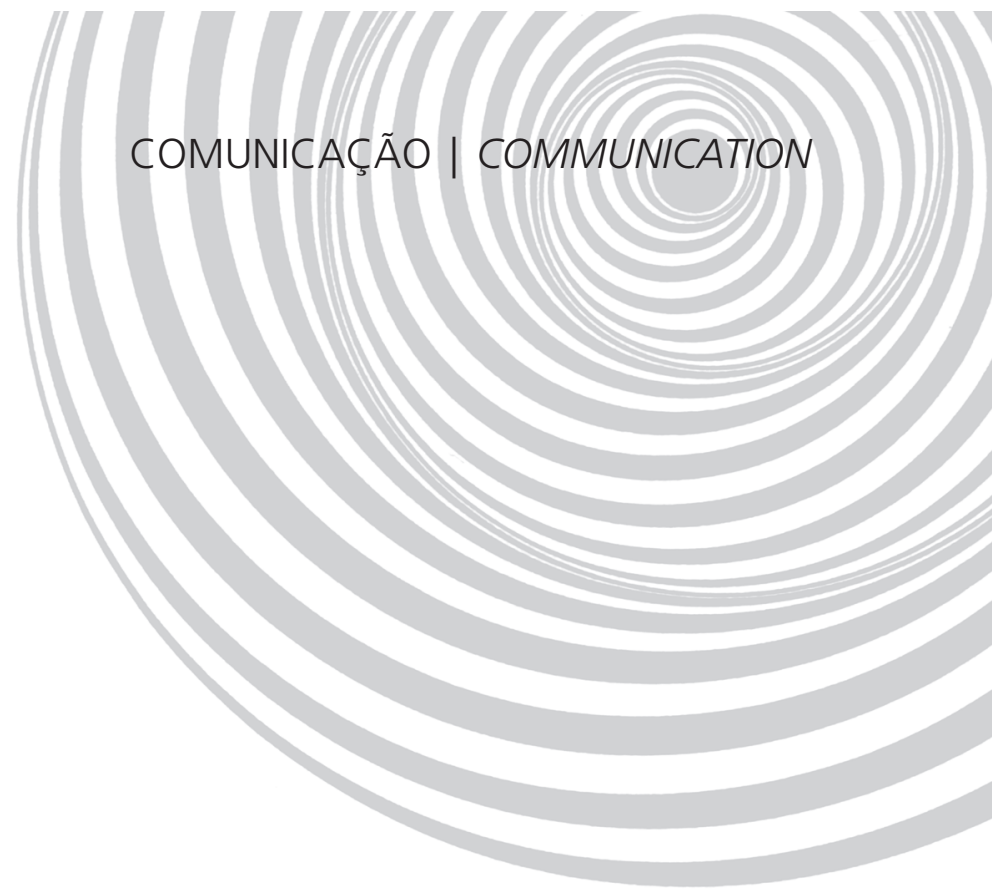

Halina Mayer Chaves ARAÚJO'

Wilma Maria Coelho ARAÚjO'

Raquel Braz Assunção BOTELHO'

Renata Puppin ZANDONADI ${ }^{1}$

RE S U M O

Esta comunicação apresenta o cenário da doença celíaca e suas implicações em hábitos, práticas alimentares e qualidade de vida de indivíduos intolerantes ao glúten. Apresenta dados importantes sobre a questão que, mundialmente, é considerada problema de saúde pública. Por ser uma doença cujo tratamento é fundamentalmente dietético, a terapia durante a transição alimentar deve ser bem conduzida pelo nutricionista para melhor adesão do paciente à dieta, considerando que a inclusão de novas práticas alimentares pode significar uma ruptura com a identidade individual e cultural: a alimentação de cada cidadão não pode ser deslocada da sociedade. Nesse contexto, a melhoria da qualidade de vida passa a ser um dos resultados esperados tanto das práticas assistenciais quanto das políticas públicas para o setor nos campos da promoção da saúde e da prevenção de doenças. A vigilância sanitária contempla as ações capazes de eliminar, diminuir ou prevenir riscos à saúde e de intervir nos problemas sanitários decorrentes do meio ambiente, da produção e circulação de bens e da prestação de serviços de interesse da saúde. O conhecimento do cenário da doença celíaca no País se justifica pela necessidade de fundamentar as ações da política de alimentação e nutrição ancoradas no conceito de alimentação saudável com ênfase na dieta isenta de glúten. Os artigos pesquisados foram selecionados nas bases de dados MedLine e SciELO, considerando o período de 1995 a 2006.

Termos de indexação: Alergias alimentares. Doença celíaca. Hábitos alimentares. Intolerância ao glúten. Qualidade de vida.

A B S T R A C T

This study presents information on celiac disease and its implications on the eating habits and practices, as well as on the quality of life, of gluten-intolerant patients. Papers published from 1995 to 2006 were searched in the MedLine and SciELO databases. This paper includes important data about this disease, which is considered a public health problem throughout the world. Since it is a disease whose treatment is essentially dietary, therapy during food transition must be well conducted by the dietician in order to improve the patient's

\footnotetext{
1 Universidade de Brasília, Faculdade de Ciências da Saúde, Departamento de Nutrição. Campus Universitário Darcy Ribeiro, Gleba A FM/FS, Asa Norte, 70910-900, Brasília, DF, Brasil. Correspondência para/Correspondence to: H.M.C. ARAÚJO. E-mail: <halina.araujo@terra.com.br>.
} 
468 | H.M.C. ARAÚJO et al.

adherence to the diet since the introduction of new food practices may mean a rupture with the individual's culture and identity: the diet of each citizen cannot be disconnected from the society in which he or she lives in. In this context, quality of life is included, as quality of life is expected to improve both because of the care provided and because of the public policies for the sector in the areas of health promotion and prevention of diseases. Sanitary surveillance contemplates the activities that are capable of eliminating, reducing or preventing health risks and intervene in the sanitation problems that are secondary to the environment, production and circulation of goods and provision of health-related services. Knowledge about celiac disease in the country is justified because of the need to base food and nutrition policies on the concept of healthy eating, emphasizing a gluten-free diet.

Indexing terms: Food allergies. Celiac disease. Eating habits. Gluten intolerance. Life quality.

\section{N T R O D U ÇÃ O}

A Doença Celíaca (DC) é uma doença autoimune desencadeada pela ingestão de cereais que contêm glúten por indivíduos geneticamente predispostos. Além do consumo do glúten e da suscetibilidade genética, é também necessária a presença de fatores imunológicos e ambientais para que a doença se expresse ${ }^{1}$. Mundialmente é considerada problema de saúde pública devido à sua prevalência, à frequente associação com morbidade variável e não específica e à probabilidade de aparecimento de complicações graves, principalmente osteoporose e doenças malignas do trato gastroentérico².

O tratamento da doença celíaca é fundamentalmente dietético. Consiste na exclusão do glúten, termo utilizado para descrever frações proteicas encontradas no trigo, centeio, cevada, aveia e em seus derivados. Para garantir uma dieta isenta de glúten, o celíaco deve sempre conhecer os ingredientes que compõem as preparações alimentares e fazer leitura minuciosa dos ingredientes listados nos rótulos de produtos industrializados. Os celíacos relatam que a oferta de alimentos sensorialmente apropriados é restrita, o que torna a dieta monótona, e que os produtos disponíveis no mercado são normalmente de alto custo.

\section{O E N ÇA C E LÍ A C A}

\section{Prevalência}

Atualmente não há dúvidas de que a doença celíaca é uma afecção mais comum no Brasil do que previamente se supunha. Ademais, assim como ocorre em outras partes do mundo, pode permanecer sem diagnóstico por prolongado período de tempo ${ }^{2}$.

A prevalência da doença celíaca entre os países e em populações europeias ou de ancestralidade europeia varia de $0,3 \%$ a $1,0 \%$; muitos casos, provavelmente, permanecem sem diagnóstico ${ }^{3}$. No Brasil, os dados estatísticos oficiais são desconhecidos; estima-se que existam 300 mil brasileiros portadores da doença, com maior incidência na Região Sudeste ${ }^{1}$. A doença celíaca é mais frequente em mulheres, numa proporção de $2: 1^{4}$, e atinge predominantemente os indivíduos de cor branca. No Brasil, devido à miscigenação racial, já foi descrita em mulatos ${ }^{5}$.

Pesquisa sobre o rastreamento de prováveis casos dessa doença entre pacientes adultos usuários dos laboratórios de análises clínicas de um hospital universitário mostrou que a prevalência nesses indivíduos é de 1:501 na forma silenciosa e clássica ${ }^{6}$. Estudo que consistiu em um rastreamento de prováveis casos de doença celíaca entre crianças usuárias do laboratório de análises clínicas no mesmo hospital apresentou uma prevalência de $1: 215^{7}$, e outro trabalho, a prevalência de 1:185 (0,54\%) em crianças componentes de um grupo de usuários de laboratório de análises clínicas de um hospital ${ }^{8}$. Esses estudos levam a crer que a prevalência da doença celíaca no Brasil, se não for similar à encontrada na Europa, em muito se aproxima.

\section{Manifestação e diagnóstico}

A doença celíaca se manifesta por meio do contato da gliadina com as células do intestino 
delgado, provocando uma resposta imune a essa fração, com a produção de anticorpos. O consumo de cereais que contêm glúten por celíacos prejudica, frequentemente, o intestino delgado, atrofiando e achatando suas vilosidades e conduzindo, dessa forma, à limitação da área disponível para absorção de nutrientes ${ }^{9}$.

Pode potencialmente afetar qualquer órgão e não apenas o trato gastroentérico. Sua eclosão e o aparecimento dos primeiros sintomas podem ocorrer em qualquer idade e variar entre indivíduos, inclusive no mesmo indivíduo em diferentes fases da doença, o que dificulta o diagnóstico ${ }^{10}$.

Richard Logan, em 1991, comparou a distribuição das formas da doença celíaca a um iceberg: os casos de apresentação sintomática corresponderiam a sua porção visível e os de apresentação assintomática, à porção submersa ${ }^{11}$. Tal fato é um obstáculo a ser superado no que diz respeito à saúde pública, pois pacientes não diagnosticados tendem a apresentar complicações relacionadas à qualidade de vida.

A doença celíaca pode ter as seguintes formas clínicas de apresentação: clássica, não clássica, latente e assintomática'. A forma clássica se manifesta principalmente nos primeiros anos de vida com sintomas como diarreia ou constipação crônica, anorexia, vômitos, emagrecimento, comprometimento variável do estado nutricional, irritabilidade, inapetência, deficit do crescimento, dor e distensão abdominal, atrofia da musculatura glútea e anemia ferropriva ${ }^{5}$.

As formas não clássicas caracterizam-se pela ausência de sintomas digestivos ou, quando presentes, ocupam um segundo plano. Apresentam-se mais tardiamente na infância. Os pacientes podem mostrar manifestações isoladas, como baixa estatura, anemia por deficiência de ferro refratária à ferroterapia oral, artrite, constipação intestinal, osteoporose e esterilidade².

A forma latente é identificada em pacientes com biopsia jejunal normal, consumindo glúten; diferencia-se das outras formas uma vez que, em outro período de tempo, tais pacientes podem apresentar atrofia subtotal dessas vilosidades intestinais, que revertem à normalidade com a retirada do glúten da dieta ${ }^{1}$.

A doença celíaca assintomática, comprovada fundamentalmente entre familiares de primeiro grau de pacientes celíacos, vem sendo reconhecida com maior frequência nas últimas duas décadas após o desenvolvimento de marcadores sorológicos específicos ${ }^{1}$. A realização de rastreamento sorológico com os anticorpos antitransglutaminase e antiendomísio é aceita como diagnóstico definitivo quando os resultados são positivos e confirmados pela biópsia intestinal, seguida pela resposta sorológica à dieta isenta de glúten ${ }^{10}$. Tal técnica permite a identificação de outras formas de manifestação da doença, além da digestiva'.

O suposto diagnóstico da doença celíaca se baseia no exame clínico, na anamnese detaIhada, na análise histopatológica do intestino delgado e na avaliação dos marcadores séricos. O diagnóstico final deve ser fundamentado na biópsia que revela vilosidades atrofiadas, alongamentos de criptas e aumento dos linfócitos intraepiteliais $^{12}$.

\section{O PAPEL DO GLÚTEN N A PRODUÇÃO DE ALIMENTOS}

O glúten é uma substância elástica, aderente, insolúvel em água, responsável pela estrutura das massas alimentícias. É constituído por frações de gliadina e de glutenina, que, na farinha de trigo, totalizam $85 \%$ da fração proteica. Forma-se pela hidratação dessas proteínas, que se ligam entre si e a outros componentes macromoleculares por meio de diferentes tipos de ligações químicas. O trigo é o único cereal que apresenta gliadina e glutenina em quantidade adequada para formar o glúten. No entanto, essas proteínas podem ainda estar presentes em outros cereais, como cevada, centeio e aveia, nas formas, respectivamente, de hordeína, secalina e avenina. 
A gliadina e a glutenina são a base da utilização da farinha de trigo na preparação industrial ou doméstica de produtos de panificação e de massas. Isso se deve à funcionalidade dessas proteínas, que determinam características importantes na aceitação dos alimentos, afetando significativamente sua qualidade sensorial. Tais propriedades resultam da habilidade que apresentam com respeito ao desenvolvimento de características sensoriais, cinestésicas, de hidratação, de atividade superficial, estrutural, dentre outras.

Esses cereais, consequentemente, podem ser adicionados durante o processamento ou o preparo de alimentos na indústria, em domicílio ou nos serviços de alimentação. É comum a adição de trigo na produção de cafés instantâneos, achocolatados em pó, sorvetes, chicletes, sopas e papas enlatadas/desidratadas, embutidos cárneos, maioneses, molhos de tomate, mostardas, iogurtes, alimentos infantis. A presença de gliadina pode ainda ocorrer pela contaminação da farinha de trigo no ambiente, pelos utensílios, pelos manipuladores de alimentos que elaboram produtos com ou sem farinha de trigo.

Piccolotto ${ }^{13}$ analisou 177 produtos industrializados disponíveis no mercado nacional. Desses, 83 eram produtos de panificação, 34 bebidas, 24 condimentos, 22 embutidos cárneos e 14 produtos desidratados. Os resultados obtidos por ensaio imunoenzimático comercial baseado num anticorpo monoclonal para $\omega$-gliadinas mostraram que o glúten esteve presente em $84 \%$ dos produtos. Dos 98 alimentos naturalmente isentos de glúten, apenas 19 (19,38\%) não apresentaram a proteína em sua composição; 4 amostras continham teores de glúten entre 0,016 e 0,046\% e apenas uma amostra apresentou teor entre 0,10\% e $0,30 \%$. Tais resultados se devem provavelmente ao fato de que, durante o processamento, produtos naturalmente isentos de glúten sofreram contaminação inaceitável aos celíacos. Segundo o Codex Alimentarius $(1995,1997)^{14}$, o limite máximo diário permitido aos celíacos é $10 \mathrm{mg}$ de gliadina.

\section{O E N ÇA CELÍACA, H Á B ITOS, PRÁT ICAS ALIMENTARES E QUALIDADE DE VIDA}

A dieta ocidental inclui muitos itens alimentícios à base de trigo. A adesão e a obediência à dieta isenta de glúten requer autodeterminação do celíaco e de seus familiares. Estudo realizado com os membros cadastrados na Associação de Celíacos do Brasil (ACELBRA) mostrou que, dentre os $91,4 \%$ participantes da pesquisa, $69,4 \%$ aderiram à dieta isenta de glúten ${ }^{15}$, estando de acordo com os dados apresentados na literatura: $65,0 \%$ e $70,0 \%{ }^{2}$.

No Canadá, pesquisa evidenciou que $88,0 \%$ dos celíacos mantêm dieta isenta de glúten ${ }^{16}$; na Índia, a dieta é seguida por $89,6 \%$ dos pacientes ${ }^{17}$. Outro estudo, também realizado no Canadá, identificou que as principais razões para a ingestão de alimentos que contêm glúten por celíacos eram: erro ou engano $(65,0 \%)$, escolha própria $(36,0 \%)$ e falta de alternativa $(27,0 \%)$. Demonstrou, ainda, que com relação ao nível de satisfação com produtos isentos de glúten, a maioria dos celíacos relatou estar medianamente ou muito satisfeita com informações apresentadas nos rótulos, disponibilidade regional dos produtos, sabor, variedade e textura. Apenas $12,0 \%$ dos celíacos entrevistados, porém, mostraram-se satisfeitos com o preço de produtos sem glúten ${ }^{18}$.

Trabalho desenvolvido com 2681 adultos celíacos, no Canadá, revelou que $44,0 \%$ dos entrevistados relataram grande ou moderada dificuldade na escolha dos componentes de sua dieta. Entre os problemas citados, destacam-se: dificuldade em determinar se os alimentos eram livres de glúten $(85,0 \%)$ e de encontrar alimentos isentos de glúten no mercado $(83,0 \%)^{18}$.

Situações como viajar, alimentar-se fora do lar e relacionar-se com amigos e familiares podem representar problemas para os celíacos e interferir, dessa forma, na sua vida social. Seguir uma dieta isenta de glúten representa mudanças no estilo de vida; 79,0\% de celíacos entrevistados numa pesquisa disseram evitar frequentar serviços de 
alimentação e 43,0\% disseram evitar viajar em função da dificuldade de encontrar produtos próprios no mercado e do risco da ingestão acidental de produtos com glúten ${ }^{18}$. Uma outra pesquisa com 253 adultos celíacos, nos Estados Unidos, revelou que a aderência à dieta isenta de glúten exercia impacto negativo sobre o ato de alimentar-se fora do lar $(86,0 \%)$ e de viajar $(82,0 \%)^{19}$.

No Brasil, com o objetivo de minimizar as dificuldades da adesão ao tratamento, surgiram as Associações de Celíacos. Em fevereiro de 1994, os pais de alguns celíacos fundaram a ACELBRA (Seção São Paulo)', que objetiva, principalmente, orientar os pacientes quanto à doença e à dieta sem glúten, assim como divulgar a doença ${ }^{20}$.

Além disso, a ACELBRA visa ainda exigir o cumprimento da Lei n 8.543 (Brasil, 1992) na área de vigilância sanitária, que obriga as indústrias alimentícias a imprimirem em caracteres destacados uma advertência nos rótulos e nas embalagens de produtos industrializados que contêm glúten ou seus derivados. Em 2003, foi publicada a Lei $n^{\circ} 10.674$, que obriga os produtos alimentícios comercializados a portarem informação sobre a presença de glúten como medida preventiva e de controle da doença celíaca. Assim, todos os alimentos industrializados deverão conter em seu rótulo, obrigatoriamente, as inscrições "contém glúten" ou "não contém glúten", conforme o caso.

Segundo a Associação dos Celíacos do Brasil ${ }^{20}$, os pacientes transgridem a dieta por vários motivos: falta de orientação relativa à doença e ao preparo de alimentos, descrença na quantidade de produtos proibidos, dificuldades financeiras, hábito do consumo de alimentos preparados com farinha de trigo, falta de habilidade culinária para o preparo de alimentos isentos de glúten.

A alimentação é componente prioritário nas sociedades contemporâneas e tem repercutido, de forma crescente, na área de saúde. A mesa é o centro das relações; simboliza organização, crítica familiar, alegrias, dissabores, novidades. Os efeitos socializantes de alimentar-se em grupo harmonizam, fortalecem vínculos, estabilizam estruturas de convívio ${ }^{21}$.
O comportamento de consumo como expressão da classe social é definido por Bourdieu como habitus, que traduz estilos de vida, julgamentos políticos, morais e estéticos. O hábito alimentar é um código que extrapola o ato de comer, podendo levar à compreensão da organização da produção econômica de uma sociedade e suas relações sociais. É por meio dos hábitos alimentares que os indivíduos, ou grupos, respondem a pressões sociais e culturais, selecionam, consomem e utilizam porções de conjunto de alimentos disponíveis ${ }^{21}$.

Por sua vez, os padrões alimentares obedecem a uma lógica em que opera, de um lado, uma estratégia de subsistência em que são maximizados os recursos e fatores dos quais dependem a reprodução da força de trabalho e a sobrevivência da família e, de outro, um sistema de conhecimento e de princípios ideológicos pelo qual se procura otimizar a relação alimento/organismo. Da conjunção desses planos resultam os padrões que caracterizam os hábitos alimentares ${ }^{21}$.

A alimentação está envolta nos mais diversos significados e as práticas alimentares incluem as etapas que vão desde os procedimentos relacionados ao preparo do alimento até o seu consumo propriamente dito. A subjetividade veiculada inclui a identidade cultural, a condição social, a religião, a memória familiar, a época ${ }^{22}$.

Para Poulain \& Proença23, os dados que permitem descrever e compreender o fenômeno alimentar podem se distribuir sobre uma sequência de elementos: as práticas observadas, as objetivadas pelos seus traços, as reconstruídas com a ajuda de instrumentos de anamnese ou rememorização, as descritas de maneira espontânea, as normas sociais expressas pelo indivíduo, as opiniões, as atitudes, os valores e as representações simbólicas.

Fome, sabor, aparência do alimento, tempo disponível ao preparo seguido da conveniência ou facilidade de compra e, em alguns casos, a condição financeira para adquiri-lo são supostamente fatores de motivação para a seleção dos alimentos ${ }^{22}$. 
Os gostos são construídos de acordo com o que a cultura estabelece como aceitável. As pessoas tendem a rejeitar sabores aos quais não estão acostumadas e permanecem restritas às preparações características de sua cultura22. Tais particularidades provavelmente justifiquem a dificuldade que indivíduos possam apresentar de adesão às novas práticas e hábitos alimentares, determinadas por situações fisiológicas específicas.

Os hábitos alimentares estão envolvidos com o sentimento dos indivíduos. Relacionam-se com valores familiares e culturais. Nesse aspecto, pode-se considerar que a restrição alimentar causa sofrimento, rompimento do afeto, perda do alimento. A experiência da doença impõe uma ruptura obrigatória de um hábito alimentar e a adesão a uma dieta isenta de glúten implica a adoção de novas práticas alimentares. Se os celíacos não transgridem a dieta, essas novas práticas podem-se tornar hábitos alimentares.

Aderir a uma dieta isenta de glúten não influencia apenas o consumo de alimentos, mas também a qualidade de vida dos pacientes.

Na área da saúde, o interesse pelo conceito de qualidade de vida é relativamente recente e decorre, em parte, dos novos paradigmas que têm influenciado as políticas e as práticas do setor nas últimas décadas ${ }^{24,25}$.

A literatura destaca que a avaliação da qualidade de vida não deve se limitar, simplesmente, a medir a presença e a gravidade dos sintomas de uma doença. Deve também ser capaz de mostrar como as manifestações de uma doença ou tratamento são experimentadas pelo indivíduo ${ }^{26}$.

As necessidades em saúde se ampliam em decorrência de um conjunto de interações de processos de natureza econômica, social, cultural, política e científico-tecnológica que não têm sido capazes de operar repercussões positivas na saúde e na qualidade de vida de amplos contingentes populacionais ${ }^{27}$

Quando se trata de produtos, processos e serviços de interesse da saúde, a complexidade da questão se amplia, pois à qualidade, à eficácia e à segurança esperadas somam-se outros elementos inerentes a um bem essencial, tais como disponibilidade, preço e acessibilidade, que não podem ser subjugados à lógica de mercado.

Os serviços de saúde e certas categorias de produtos sob controle sanitário são indispensáveis à consecução do direito à saúde, pelo quais as ações de vigilância sanitária ultrapassam o âmbito da defesa e proteção da saúde do consumidor para abarcar a população como um todo. Indo além, a própria questão do consumo reporta-se à reflexão sobre o direito de ser consumidor de bens e serviços necessários a uma boa qualidade de vida, o que não tem sido equacionado para todos os segmentos nas sociedades atuais ${ }^{28,29}$, inclusive para os portadores de doenças.

\section{CONSIDERAÇÕES FINAIS}

Nos celíacos, os quadros de desnutrição e hipernutrição são comuns; a desnutrição é decorrente da má absorção de nutrientes e da dificuldade da ingestão alimentar em função dos sintomas apresentados. Nos indivíduos em tratamento, o quadro de hipernutrição se deve à maior absorção de nutrientes, decorrente da possível melhora desses sintomas, que estimula maior ingestão alimentar e ao fato de os alimentos para celíacos normalmente apresentarem maior quantidade de lipídios em sua composição.

Em função do tratamento para essa doença ser unicamente dietético e da dificuldade da exclusão dos cereais que contêm glúten da dieta, observa-se a importância do profissional de Nutrição na avaliação do estado nutricional, na orientação relativa à escolha, ao preparo dos alimentos e à contaminação por glúten na etapa de preparo ou distribuição do alimento e nas orientações relativas à deficiência de absorção de macro e micronutrientes. Verifica-se também a necessidade de acompanhamento constante para avaliar a adequação da ingestão dietética, presença de transgressões - voluntárias ou não - e sinais de comprometimento nutricional, que são fatores 
determinantes na qualidade de vida do paciente celíaco.

O nutricionista, assim, estimula a adesão ao tratamento, evita a monotonia e acompanha a ingestão alimentar. Além disso, deve estar atento para que haja uma transição alimentar não traumática para melhor adesão à dieta.

Os celíacos, além dos fatores supracitados, apresentam dificuldades relativas à alimentação fora do lar em função do desconhecimento da população sobre esse assunto. É comum observar-se nos serviços de alimentação e em unidades produtoras de refeição a utilização de farinha de trigo em preparações que tradicionalmente não deveriam contê-la, como peixe grelhado, feijão, molhos, o que dificulta a adesão ao tratamento e, consequentemente a melhoria da qualidade de vida desses pacientes. Deve-se, portanto, conscientizar o setor de alimentação fora do lar para que se preocupe e faça a inclusão de celíacos.

Considerando ainda que a vigilância sanitária contempla as ações capazes de eliminar, diminuir ou prevenir riscos à saúde e de intervir nos problemas sanitários decorrentes do meio ambiente, da produção e circulação de bens e da prestação de serviços de interesse da saúde e constitui-se um subsetor específico da saúde pública com uma face mais complexa, ela passa a compor o elenco dos direitos fundamentais dos indivíduos no seu amplo espectro de ação.

Nesse aspecto, verifica-se a necessidade de melhor caracterizar o cenário da DC, considerando que a natureza das ações de vigilância sanitária é eminentemente preventiva, devendo atuar sobre fatores de riscos e danos e seus determinantes associados a produtos. Deve-se atentar para o fato de que a alimentação de cada cidadão não pode ser deslocada da sociedade. A inclusão de novas práticas alimentares pode significar uma ruptura com a identidade individual e cultural.

\section{OLABORADORES}

H.M.C. ARAÚJO participou da concepção do projeto, da realização das pesquisas bibliográficas, redigiu e discutiu o manuscrito. W.M.C. ARAÚJO participou da concepção e da discussão e da redação do artigo. R.B.A. BOTELHO e R.P. ZANDONADI participaram das discussões do artigo.

\section{REFERÊ NCIAS}

1. Sdepanian VL, Morais MB, Fagundes-Neto U. Doença celíaca: a evolução dos conhecimentos desde sua centenária descrição original até os dias atuais. Arq Gastroenterol. 1999; 36(4):244-57.

2. Pratesi R, Gandolfi L. Doença celíaca: a afecção com múltiplas faces. J Pediatr. 2005; 81:357-8.

3. Catassi C, Fornaroli F, Fasano A. Celiac disease: from basic immunology to bedside practice. Clin Appl Immunol Rev. 2002; 3(2):61-71.

4. Gandolfi L, Pratesi L, Cordoba JC, Tauil PI, Gasparin $\mathrm{M}$, Catassi C. Prevalence of celiac disease among blood donors in Brazil. Am J Gastroenterol. 2000; 95:689-92.

5. Rauen MS, Back JCV, Moreira EAM. Doença celíaca: sua relação com a saúde bucal. Rev Nutr. 2005; 18(2):271-6. doi: 10.1590/\$1415-527320050002 00011.

6. Garcias SG. Rastreamento de prováveis casos de doença celíaca entre pacientes adultos usuários de laboratórios de análises clínicas em Hospital Geral, no Distrito Federal [dissertação]. Brasília: Universidade de Brasília; 1999.

7. Modelli ICS. Rastreamento de prováveis casos de doença celíaca entre crianças usuárias de laboratório de análises clínicas em Hospital Geral, no Distrito Federal [dissertação]. Brasília: Universidade de Brasília; 2001.

8. Pratesi R, Gandolfi L, Garcia SG, Modelli IC, Almeida PL. Prevalence of coeliac disease: unexplained age-related variation in the same population. Scand J Gastroenterol. 2003; 38:747-50.

9. Thompson $T$, Dennis M, Higgins LA, Lee AR, Shavrett MK. Gluten-free diet survey: are Americans with celiac disease consuming recommended amounts of fibre, iron, calcium and grain foods? J Hum Nutr Diet. 2005; 18:163-9.

10. Pratesi R, Gandolfi L. Doença celíaca: a afecção com múltiplas faces. J Pediatr. 2005; 81(5): 357-8.

11. Sdepanian VL, Morais MB, Fagundes-Neto U. Doença celíaca: a evolução dos conhecimentos desde sua centenária descrição original até os dias atuais. Arq Gastroenterol. 1999; 36(4):244-57.

12. Brasnki D, Troncone R. Celiac disease: a reappraisal. J Pediatr. 1998, 133:181-7. 
13. Picolloto FMBB. Determinação do teor de glúten por ensaio imunoenzimático em alimentos industrializados [tese]. São Paulo: Faculdade de Engenharia de Alimentos; 2002.

14. Food and Agriculture Organization of the United Nations. Codex Alimentarium Commission. Proposed draft revised standard for gluten-free foods (At Step 5 of the Procedure). In: Food and Agriculture Organization of the United Nations. Codex Alimentarium Commission. Codex alimentarius. Report of the 20th Session of the Codex Committee on Nutrition and Foods for Special Dietary Uses; Bonn-Bad 1997 October 7-11; Godesberg, Germany. Roma: FAO; 1997. p.5-6, 33-41. (ALINORM 97/26).

15. Sdepanian VL, Morais MB, Fagundes-Neto U. Doença celíaca: avaliação da obediência à dieta isenta de glúten e do conhecimento da doença pelos pacientes cadastrados na Associação dos Celíacos do Brasil (ACELBRA). Arq Gastroenterol. 2001; 38(4):232-9.

16. Cranney A, Zarcadas M, Graham ID, Switzer C. The Canadian celiac health survey: the Ottawa chapter pilot. In: Butterworth JR, Banfield LM, Iqbal TH, Cooper BT. Factors relating to compliance with a gluten-free diet in patients with celiac disease: comparison of with Caucasian and South Asian patients. Clin Nutr. 2004; 23(5):1-8.

17. Sood A, Midha V, Sood N, Malhotra V. Adult celiac disease in Northern India. In: Butterworth JR, Banfield LM, Iqbal TH, Cooper BT. Factors relating to compliance with a gluten-free diet in patients with celiac disease: comparison of with Caucasian and South Asian patients. Clin Nutr. 2004; 23(5):1-8.

18. Lamontagne P, West GE, Galibois I. Quebecers with celiac disease: analysis of dietary problems. Can J Diet Pract Res. 2001; 62(4):175-80.

19. Zarcadas M, Case $S$. The gluten-free diet: its impact on the quality of life of adult patients with celiac disease. In: Zarcadas M, Case S. Celiac disease and the Gluten-free Diet. Top Clin Nutr. 2005; 20(2): 127-38.

20. Associação de Celíacos do Brasil. [acesso 2004 nov 15]. Disponível em: <http://www.acelbra.org.br>.
21. Botelho RBA. Culinária regional: o Nordeste e a alimentação saudável [tese]. Brasília: Universidade de Brasília; 2006.

22. Garcia RWD. Práticas e comportamento alimentar no meio urbano: um estudo no centro da cidade de São Paulo. Cad Saúde Pública. 1997; 13(3): 455-67.

23. Poulain JP, Proença RPC. Reflexões metodológicas para o estudo das práticas alimentares. Rev Nutr. 2003; 16(4):365-86. doi: 10.1590/\$1415-527320 03000400011 .

24. Rogerson RJ. Environmental and health-related quality of life: conceptual and methodological similarities. In: Seidl EMF, Zannon CMLC. Qualidade de vida e saúde: aspectos conceituais e metodológicos. Cad Saúde Pública. 2004; 20(2):580-8.

25. Schuttinga JA. Quality of life from a federal regulatory perspective. In: Seidl EMF, Zannon CMLC. Qualidade de vida e saúde: aspectos conceituais e metodológicos. Cad Saúde Pública. 2004; 20(2):580-8.

26. Teixeira-Salmela LF, et al. Adaptação do perfil de saúde de Nottingham: um instrumento simples de avaliação da qualidade de vida. Cad Saúde Pública. 2004; 20(4):905-14.

27. Organización Panamericana de la Salud. La crisis de la salud pública: Reflexiones para el debate. In: Costa EA. Vigilância sanitária e proteção da saúde. [acesso 2006 maio 15]. Disponível em: <http://www. saude.ba.gov.br/conferencia>.

28. Costa, EA. Vigilância Sanitária e Proteção da Saúde. [acesso 2006 jun 1]. Disponível em: <http://www. saude.ba.gov.br/conferencia>.

29. Mancuso RC. Interesses difusos: conceito e legitimação para agir. In: Costa, EA. Vigilância sanitária e proteção da saúde. [acesso 2006 jun 1]. Disponível em: <http://wwww.saude.ba.gov.br/conferencia>.

Recebido em: 16/4/2008

Versão final reapresentada em: 7/10/2009 Aprovado em: 17/3/2010 